\title{
The Venus Phosphine Debate Continues
}

\author{
Researchers remain divided on the possible discovery of phosphine on \\ Venus, a finding that could have implications for whether life resides on \\ this nearby planet.
}

By Katherine Wright

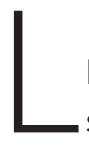

ife might exist beyond Earth, at least so claimed headlines across the globe in September touting possible signs of a gas called phosphine in the atmosphere of Venus. As all of Earth's naturally occurring phosphine is produced by microbial life, the astronomical observations of the gas, reported by Jane Greaves at the University of Cardiff, UK, and colleagues, opened the door to speculation of microscopic Venusians floating in the planet's clouds. But a flurry of papers appeared soon after on the arXiv preprint server questioning

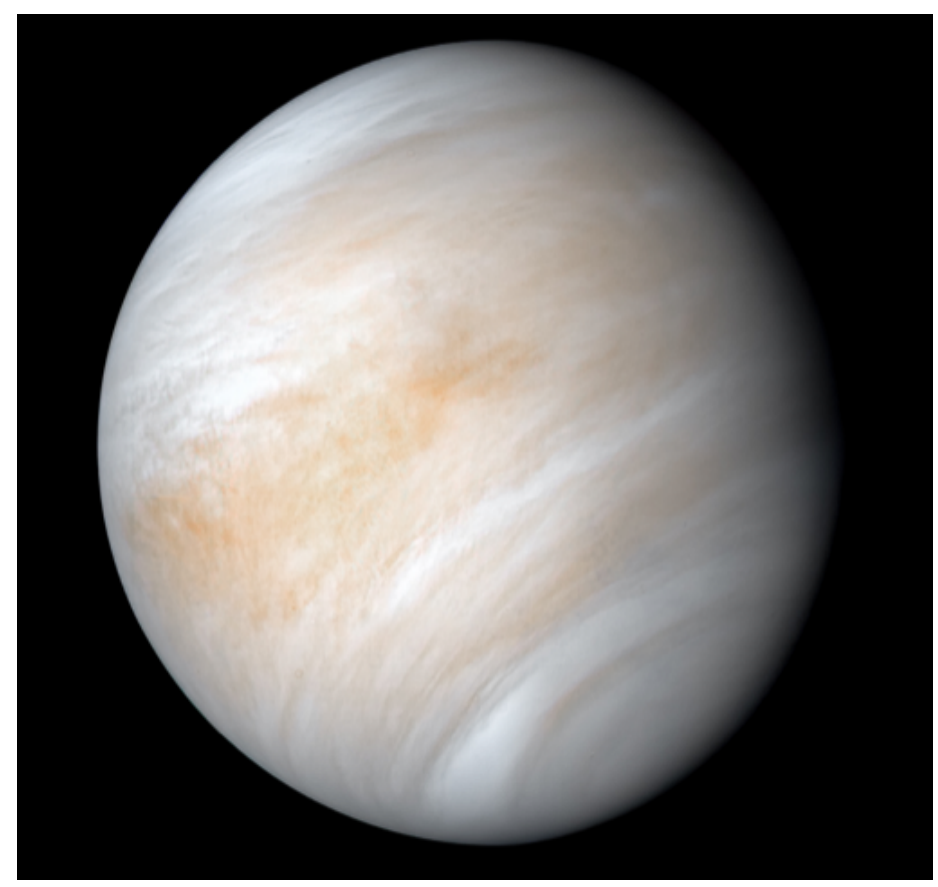

Credit: NASA/JPL-Caltech the result. This debate received airtime earlier this month at the 2020 American Geophysical Union Fall Meeting, where Greaves presented an updated analysis of the team's data, which continue to point to the existence of phosphine on the planet.

The data indicate a 5-sigma detection, which is the usual standard for a scientific discovery. "If it's good enough for the Higgs boson, it's good enough for us," Greaves said.

One of the ways that astrobiologists search for extraterrestrial life is by collecting the light scattered from a distant planet and scouring it for the spectral fingerprints of biomarkers-molecules that on Earth have a biological origin. Phosphine $\left(\mathrm{PH}_{3}\right)$ is one such molecule; it is produced on Earth by bacteria living in oxygen-deficient environments, such as swamps, sewers, and human guts. Researchers have long suggested that the clouds of Venus could provide a similar environment, where anaerobic lifeforms would have access to water and sunlight while remaining safe from the planet's life-obliterating, $470^{\circ} \mathrm{C}$ surface. Taken with this idea, Greaves and her colleagues decided in 2017 to take a look. "It seemed worth investing a few hours of telescope time to search for phosphine," Greaves said.

For their measurements, the team first turned to the James Clerk Maxwell Telescope (JCMT) in Hawaii. The telescope detects submillimeter wavelengths, which coincide with an electronic transition of phosphine. The team's JCMT observations indicated that $\mathrm{PH}_{3}$ is present in Venus's atmosphere at a concentration of about 20 parts per billion (ppb)-for context, Earth's atmosphere contains a global 
average of a few parts per trillion. "To our astonishment, we had what looked like a detection," Greaves said.

The team followed this measurement by further observations with JCMT, as well as with the Chilean Atacama Large Millimeter/submillimeter Array (ALMA), which operates in the same spectral regime but has greater spectral resolution. Those measurements all found the same $\mathrm{PH}_{3}$ transition. Ideally, Greaves said, her team would have sought supporting evidence from a third telescope that captured a different $\mathrm{PH}_{3}$ transition, but telescopes that do so don't yet exist. "So, in the interest of transparency, we published the data." And that is where the controversy began.

The debate largely centers on the team's data analysis. The absorption line of $\mathrm{PH}_{3}$ measured by ALMA is hidden in an extremely wiggly spectral background. To subtract that background, the team had to fit the data with a 12th-order polynomial. Such a polynomial has a huge number of undefined variables, allowing many opportunities for fit errors to creep in. Greaves noted, however, that this polynomial is commonly used by ALMA to remove instrumental artifacts. Yet, an analysis of Greaves' data by Geronimo Villanueva, of NASA Goddard Space Flight Center, and colleagues uses a different calibration method with lower-order polynomials and finds no signature of $\mathrm{PH}_{3}$. The spectrum is so wiggly in this region that identifying lines pushes the boundaries of the telescope instruments, Villanueva said.

Instead Villanueva-and others-think that the signal comes from sulfur dioxide $\left(\mathrm{SO}_{2}\right)$, which has a spectral peak very close to that of phosphine. $\mathrm{SO}_{2}$ has previously been found to exist in abundances of about 100 ppb in Venus's atmosphere, and Villanueva's analysis indicates that an $\mathrm{SO}_{2}$ level of just half that concentration could drop the phosphine signal in Greaves' analysis to below 3 sigma, which would no longer imply strong evidence for the gas.

" $\left[\mathrm{SO}_{2}\right]$ is a plausible alternative," Villanueva said. Data from other experiments, including NASA's Infrared Telescope Facility (IRTF) in Hawaii and ESA's Venus Express spacecraft, also fit with an $\mathrm{SO}_{2}$ explanation. "Another detection is definitely needed to confirm the reality of the [phosphine] identification," said Therese Encrenaz of the LESIA Observatory of Paris. She and her colleagues restudied measurements from the TEXES instrument at IRTF, which is used to monitor $\mathrm{SO}_{2}$ on Venus. She said lines consistent with the presence of $\mathrm{PH}_{3}$ are detectable in data taken from March 2015, but the molecule would have to be higher in the atmosphere than suggested by Greaves' data and also have different abundances.

Greaves and her colleagues have taken on board these criticisms and issues-as well as others-and reanalyzed their ALMA data, releasing an updated paper a month ago on the arXiv. Their new analysis continues to find a phosphine signal, with a 4.8-sigma confidence level. The updated results, however, indicate that the signal comes from a lower phosphine concentration of 1 to $4 \mathrm{ppb}$ when averaged over the whole planet, with a peak of 5 to 10 ppb.

Despite the disagreements, the scientists remained friendly in their discussions. Villenueva, for example, congratulated Greaves for how she and her team held onto their initial results until they had confirmation from a second instrument. "They did their due diligence," he said. "I think that this is highly commendable."

Correction (5 January 2021): A previous version incorrectly described potential lifeforms from Venus as "Venetians." Also, a description of the ALMA data's signal-to-noise ratio was removed to avoid a possible confusion.

Katherine Wright is a Senior Editor for Physics. 